\title{
My Virtual Colleague: A State-of-the-Art Analysis of Conversational Agents for the Workplace
}

\author{
Shengjia Feng \\ SAP SE and Technische Universität Darmstadt \\ shengjia.feng@sap.com
}

\author{
Peter Buxmann \\ Technische Universität Darmstadt \\ buxmann@is.tu-darmstadt.de
}

\begin{abstract}
Conversational interfaces at the workplace are not a new idea, but it is only the recent technological advancements that turned what was once a vision into near-future reality. Improved reliability and accuracy enable conversational systems to be used in higher stake environments, such as the workplace. In this work, we perform a literature review on concepts proposed to incorporate Conversational Agents (CA) into the workplace. We found 29 workplace CAs designed for workers that contribute to eight different application domains. Based on the studies of these CAs, we compiled a list of aspects to be considered when designing such CAs and identified starting points for further research.
\end{abstract}

\section{Introduction}

Science-fiction film makers once envisioned systems in future workplaces to be operated through natural language commands [1]. Although the fully conversational workplace was still a vision for the far future, the first conversational agents (CA) ELIZA [2] and Parry [3] were presented soon after. Today, over 50 years later, the film makers vision could become reality with the emergence of CAs in the form of Siri, Alexa or Google Assistant. However, common CAs like these are mostly used for low-stake purposes such as finding a navigation route or switching lights in a smart home. Despite their popularity in private environments, concepts for CAs at the workplace are just gaining attention in the recent years [4, 5].

As the development of CAs evolves rapidly, we aim to compile an up-to-date overview of workplace CAs (WCAs) that have been proposed in scholarly research which leads to our first research question:

RQ1: What are the state-of-the-art WCA concepts?

Based on our findings, our second research question targets the design of WCAs. Due to the novelty of this type of CA, we neither expect a large number of results nor extensive studies, therefore we aim to extract general aspects rather than defined design principles:

RQ2: Which aspects need to be taken into consideration in the design of a WCA?

In order to answer these research questions, we perform a Systematic Literature Review (SLR) [6]. The remainder of this paper is structured as follows: In Section 2 we introduce the different types and dimensions of CAs. Then, we explain our SLR process in Section 3 and present our results in Section 4 Section 5 and Section 6 conclude this paper by discussing our findings, the limitations and starting points for further research.

\section{Conversational Agents}

CAs are software programs that are capable of "interpret[ing] and respond[ing] to statements made by users in ordinary natural language" [7] and should not be confused with the more general term of bots which are mostly non-conversational "automata used for background tasks" [8]. Common synonyms for CAs are chatbots, conversational (artificial) intelligence (with focus on technical aspects, e.g. [9]) or conversational (user) interface (with focus on user interaction, e.g. [10, 11]). Initially proposed by Alan Turing in 1950 [12], early CAs like ELIZA [2] and Parry [3] were presented in the 1960s and 70s, based on handcrafted rules to simulate human behavior. Although the early day CAs were perceived as convincing [13], the technological advancements in the last decade enabled CAs to become sufficiently intelligent to actually be useful [14].

There are many taxonomies proposed in scholarly research to differentiate and classify CAs. Table 1 summarizes several common dimensions: Domain Breadth and Depth are the total number of topics and the degree of detail supported by the CA. Session Length includes two values, the designated number of conversation turns per session (CPS) and whether the intent is to minimize or maximize the session length. Emotionality indicates whether a CA is capable of 


\begin{tabular}{|l|l|c|}
\hline Dimension & Example Values & Sources \\
\hline $\begin{array}{l}\text { Domain } \\
\text { Breadth }\end{array}$ & $\begin{array}{l}\text { General Purpose, } \\
\text { Domain-Specific; } \\
\text { Open, Closed }\end{array}$ & {$[17,18]$} \\
\hline Domain Depth & Shallow, Deep & {$[8]$} \\
\hline $\begin{array}{l}\text { Session } \\
\text { Length }\end{array}$ & $\begin{array}{l}\text { Number of CPS, } \\
\text { Max/Min CPS }\end{array}$ & {$[8,[9]$} \\
\hline Goal Direction & $\begin{array}{l}\text { Goal-Oriented, } \\
\text { Non-Goal-Orient. }\end{array}$ & {$[18]$} \\
\hline Emotionality & $\begin{array}{l}\text { Emotional, } \\
\text { Non-emotional }\end{array}$ & {$[18]$} \\
\hline Representation & $\begin{array}{l}\text { Disembodied, } \\
\text { Embodied }\end{array}$ & {$[18]$} \\
\hline Modalities & $\begin{array}{l}\text { Speech, Text, } \\
\text { Multi-Modal }\end{array}$ & {$[17,18]$} \\
\hline
\end{tabular}

Table 1. Dimensions of Conversational Agents

showing emotions including being empathetic and act accordingly. Lastly, the dimensions Representation and Modalities apply to the instantiation of the CA, that is, the amount of human characteristics (e.g. visualization as a human being, physical embodiment as a robot) and the input and output interfaces the CA uses to interact with the user, respectively.

CAs are often divided into three major categories (e.g. [8]): Intelligent (Personal) Assistants (IPAs), Goal-/Task-Oriented CAs (GCAs) and Virtual Companions (VCs). IPAs like Sir 1 and Google Assistan ${ }^{2}$ can perform a large range of simple actions, most of which are completed with very few CPS with the goal to minimize this number. Same optimization goal is valid for GCAs, however, they only support a narrow range of functions that require a higher CPS by the nature of the task (e.g. placing an order). In contrast, VCs are designed to maximize CPS to maintain an interesting conversation for as long as possible. Common use cases for virtual companions are entertainment purposes (e.g. $Z \alpha^{3}$ and Cognitive Behavioral Therapy (e.g. Woebot [15], Wysa [16]).

\section{Review Method}

In this section, we describe the method used for our SLR in detail, following the plea for more rigor

\footnotetext{
${ }^{1}$ https://www.apple.com/siri

${ }^{2}$ https://assistant.google.com

${ }^{3}$ https://www.zo.ai
}

in literature reviews [20]. To ensure replicability, we present our search process in detail.

We conducted exploratory searches in Google Scholar to retrieve established synonyms for our central terms "CA" and "workplace". For CA, we selected "chatbot" and the combinations of "conversational" with "bot", "agent", "AI", "UI", "intelligence" and "interface". For "workplace", we used "workspace" and "workstation" as synonyms and excluded the term "work" due to its ambiguous meaning. Our final search query therefore was (("conversational" ("agent" OR "UI" OR "AI" OR "interface" OR "intelligence" OR "bot")) OR "chatbot") AND ("workplace" OR "workspace" OR "workstation"). We executed separate searches for each combination and used the duplicate detection function in Zotero to remove duplicates. We limited our SLR to publications from year 2010 or later due to the significant technological advances in the past decade [14]. Only peer-reviewed, and, due to language barriers, only publications in English and German were included.

Our systematic search in the selected databases listed in Figure 1 yielded 338 unique publications complying to the inclusion criteria. In order to include relevant publications not matching the keywords, we manually scanned the contents of the AIS senior scholars baske 4 as well as relevant journals and conferences on the Human-Computer Interaction field selected from the Google Scholar h5-index ranking 5 with the same inclusion criteria. Thereby, we added 30 additional results, totalling at 368 publications. In the first step, we sorted out any publications that do not contribute a WCA concept, i.e. a CA concept which is either used by a working person (Cat. I) or which is not used by a working person, but does impact the work of another person who is not necessarily involved in the user-CA dialogue (Cat. II). After categorizing the remaining publications into these two categories, we performed a forward and backward search on the Cat. I publications as they are more relevant to our research goal. In total, we found 35 publications with Cat. I WCAs and 46 publications with Cat. II WCAs. A summary of our search and filtering process is provided in Figure 1.

\section{Results}

In this section, we describe our observations in our result set, then we summarize and classify the WCA concepts. Eventually, we summarize design aspects and other findings from those WCA concepts. Based on the 81 publications in our result set, we identified 29 WCAs

\footnotetext{
${ }^{4}$ https://aisnet.org/page/SeniorScholarBasket

${ }^{5}$ http://bit.ly/2WW1Vep
} 


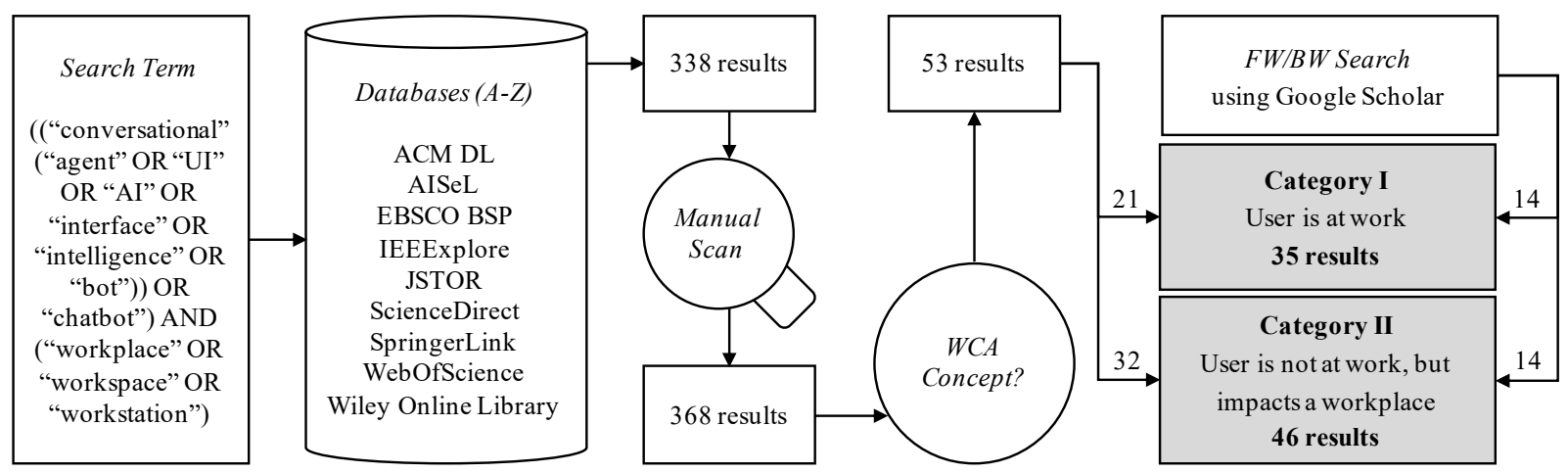

Figure 1. Our Systematic Literature Review Process

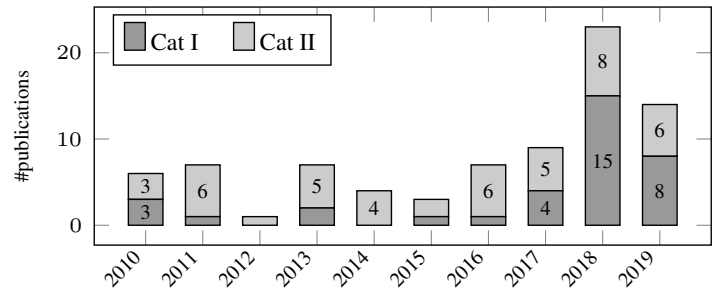

Figure 2. Distribution of Publications over Time

for workers in eight application domains and three main application domains of WCAs for non-workers.

\subsection{Descriptive Results}

In our final set of publications we observe a massive peak in the number of publications in year 2018 as depicted in Figure 2. This supports our claim that it is only the advent of WCAs, facilitated by recent technological advancements and subsequent possibilities. Although there is a drop in the number of publications in year 2019, it should be noted that this SLR was conducted in April/May 20196, thus with more than half of the year still upcoming. The U.S. and the U.K. lead the number of papers with at least one author affiliated to an institution in those countries with a total of 38 and 12 publications out of all 81 publications, respectively. This is not surprising: Many leading tech companies reside in the U.S. and there is interest in WCAs due to its high potential in commercial value. In addition, the skew towards English-speaking countries can be explained by the research efforts invested into modules like Natural Language Processing, which again, are often driven by large tech enterprises. Nonetheless, the total of 25

\footnotetext{
${ }^{6}$ Note: During revision of this paper in September 2019, a few additional papers published after April/May 2019 were added through a new forward/backward search.
}

countries from all over the world indicate the emerging interest in WCAs generally.

\subsection{Classification of WCAs}

Next to the dimensions used for classification of CAs in general, we realized during this SLR that additional dimensions are needed to classify WCAs properly. In particular, there is the productive-collaborative aspect of WCAs and the workplace it is designed for. Therefore, we use a combination of different dimensions to classify the WCAs in the following:

- General CA Dimensions are dimensions typically used for all types of CAs, as listed in Table 1. These include: Domain Depth (DD) and Breadth (DB), Session Length (SL), Goal Direction (GD), Emotionality (EMO), Representation (REP) and Modalities (MOD).

- Collaborative Dimensions are additional dimensions specific to a human-CA collaborative setup. These include: Role (RO - Faciliator, Peer, Expert), No. of Humans (NH - Individual, Team, Crowd), NCA - No. of CAs (Single, Multiple), as proposed by Bittner et al. [18]

- Workplace Dimensions are additional dimensions for the particular operating place of the WCA. These include: Impact (IMP - Decision Making, Control, Productivity, Social Interaction, Job Enhancement, Work Environment) and Type of Worker (WO - Managers, Professionals, Clericals/Technicians), as proposed by Kraemer and Danziger [21] for information workers. Due to the small amount of relevant WCAs for manual workers, we proposed to add "Manual Worker" as another value in the WO dimension without further differentiation. 


\subsection{WCAs for Workers}

We observed that among the 29 WCAs for workers (WWCAs), i.e. WCAs where the target audience are people at their respective work and workplace, the degree to which the added human characteristics of a CA were made use of varies immensely. However, this is also reasoned by the variety of application domains, of which we identified eight, as each case demands different CA characteristics. Table 2 provides an overview of the WWCAs and their classification.

Working Tool. The most common application domain among our WWCAs is to use the WCA as a tool for specific work purposes. Examples for this are Ava [22] and Iris [23], two WCAs for data scientists to perform statistical analyses. Similarly, MSRBot [24] provides insights into software code repositories by executing analytic searches. Isa [25] automatically detects customer contexts for customer service agents and provide relevant information for the agent to use, therefore being a valuable tool for seasonal workers. For neuroscience labs [26], 3D modelers [27] and bio labs [28], WCAs have been proposed to provide respective just-in-time assistance to tackle work challenges.

Information Retrieval. WCAs are often used as easy-to-use interfaces for search engines as missing information in a search query can be inquired in a natural dialogue to specify the desired information. Therefore, information retrieval WCAs are often proposed in the form of an anthropomorphised information desk agent, e.g. for anti-bullying information [29, 30], HR FAQ [31], construction safety information [32]. However, pragmatic WCAs without anthropomorphism are also proposed, e.g. to access building information [33] or to improve the accessibility of organizational information [34]. A special case is proposed in FarmChat [35] where the information provided by the WCA is respected as expert advice rather than retrieval of existing knowledge.

Routine Assistance. Similar to the working tools, Routine Assistance WCAs assist users in their work to become more productive. However, as the name indicates, their focus is on executing complex, but repeating routines that are rather administrative and/or auxiliary than an actual part of the role. This applies to meeting scheduling [36], administrative tasks accompanying courses [37], plug management in the office [38], personal and group task management [39] or incident reporting [40].

Virtual Colleague. The virtual colleague is an application domain where a strong anthropomorphism is desired as the WCA should represent a human being. In [41], the (user-programmed) WCA acts as an embodied co-presenter and in [42], the WCA represents a new hire mentor, both providing information and sending reminders and news proactively. A different application of a virtual colleague is to relieve solitude and prevent driver fatigue by conducting conversations with a truck driver during driving [43, 44, 45].

Work Environment. For the particular group of crowdworkers, WCAs have been proposed to be used as a mobile and intuitive interface [46] or, for them to actually work "inside" a CA as their workplace, providing crowd human intelligence to mimic a $\mathrm{CA}$ [47, 48]. It can be debatable whether the latter can be classified as a WCA, however, as there are workers involved in this WCA, we decided to sort it into this application domain.

Decision Making. Similar to human capabilities, WCAs can act as a mediator for group decisions (e.g. for group lunches [49]), or multiple WCAs can represent different steps of thought to assist the user in more complex decision making [50, 51].

Reflection and Control. Kocielnik et al. proposed the usage of a WCA to enable users to log a work journal for reflection purposes [52]. While UpTime [53] is a system to help users to cut out distractions, the CA element provides a possibility for the user to reflect on whether they really need to view the blocked page.

Team Creation. Lastly, a WCA can also help creating teams by analyzing member candidates in a psychological manner to identify matching profiles as proposed by Tseng et al. [54].

\subsection{WCAs for Non-Workers}

WCAs for non-workers are WCAs of which the main target audience are not working people. However, they are designed to be operated at one's workplace and thus influence the work and workplace of a person different than the user. The most prevalent goals of a WCA are to support or even replace human work. Therefore, we present selected and important application domains in more detail in the following.

In the area of health care several applications were proposed, such as a virtual discharge nurse [55, 56], as a counsellor for mental health issues [57, 58] and stress relief [59], as a trustworthy partner to talk about sensitive topics [60], to facilitate scalable depression and suicidal prevention [61,62], to enable self-reflection and behavior change [63, 64] or to support care and monitoring for elderly people [65, 66]. In all of these cases, the main user is not a working person, but rather a patient. However, successful establishment of either of these systems can greatly impact the work of the medical 


\begin{tabular}{|c|c|c|c|c|c|c|c|c|c|c|c|c|c|}
\hline Source & App. Domain & DD & DB & SL & GD & EMO & REP & MOD & RO & NH & NCA & IMP & wo \\
\hline 22 & Working Tool & D & $\mathrm{N}$ & MIN & NGO & NEMO & DEMB & $\mathrm{T}$ & $\mathrm{F}$ & IND & SING & PROD & PRO \\
\hline 23 & Working Tool & D & $\mathrm{N}$ & MIN & NGO & NEMO & DEMB & $\mathrm{T}$ & $\mathrm{F}$ & IND & SING & PROD & PRO \\
\hline 24 & Working Tool & $\mathrm{D}$ & $\mathrm{N}$ & MIN & NGO & NEMO & DEMB & $\mathrm{T}$ & $\mathrm{F}$ & IND & SING & PROD & PRO \\
\hline 25 & Working Tool & $\mathrm{D}$ & $\mathrm{N}$ & MIN & NGO & NEMO & DEMB & $\mathrm{T}$ & $\mathrm{F}$ & IND & SING & PROD & $\mathrm{C} / \mathrm{T}$ \\
\hline 26 & Working Tool & $S$ & $\mathrm{~N}$ & MIN & GO & NEMO & DEMB & $\mathrm{T}$ & $F$ & IND & SING & PROD & PRO \\
\hline 27 & Working Tool & $\mathrm{D}$ & $\mathrm{N}$ & - & NGO & NEMO & DEMB & $\mathrm{T}$ & $\mathrm{F}$ & IND & SING & PROD & PRO \\
\hline 28 & Working Tool & $\mathrm{S}$ & $\mathrm{N}$ & - & NGO & NEMO & DEMB & $S$ & $\mathrm{~F} / \mathrm{P}$ & IND & SING & PROD & PRO \\
\hline \begin{tabular}{|l|l|}
29 & 30 \\
\end{tabular} & Info. Retrieval & $\mathrm{D}$ & $\mathrm{N}$ & MIN & GO & NEMO & $\mathrm{DE}$ & $\mathrm{T}, \mathrm{H}$ & $\mathrm{F} / \mathrm{P}$ & IND & SING & WE & ALL \\
\hline 31 & Info. Retrieval & $\mathrm{S}$ & $\mathrm{B}$ & MIN & NGO & NEMO & DEMB & $\mathrm{T}$ & $\mathrm{F}$ & IND & SING & PROD & INFO \\
\hline 32 & Info. Retrieval & $\mathrm{S}$ & $\mathrm{B}$ & MIN & NGO & NEMO & EMB & $\mathrm{T}, \mathrm{S}$ & $\mathrm{F}$ & IND & SING & PROD & ALL \\
\hline 33 & Info. Retrieval & $\mathrm{S}$ & $\mathrm{N}$ & MIN & NGO & NEMO & DEMB & $\mathrm{T}$ & $\mathrm{F}$ & IND & SING & CTRL & ALL \\
\hline 34 & Info. Retrieval & $\mathrm{S}$ & B & MIN & NGO & NEMO & $\mathrm{DE}$ & $\mathrm{T}$ & $\mathrm{F}$ & IND & SING & WE & INFO \\
\hline 35 & Info. Retrieval & $\mathrm{D}$ & $\mathrm{B}$ & MIN & GO & NEMO & $\mathrm{DE}$ & $\mathrm{T}, \mathrm{S}$ & $E$ & IND & SING & PROD & PRO \\
\hline 36 & Routine Assist. & $\mathrm{S}$ & $\mathrm{N}$ & MIN & GO & NEMO & $\mathrm{DE}$ & $\mathrm{T}$ & $F$ & TEAM & SING & PROD & INFO \\
\hline 37 & Routine Assist. & & & & & & & & & & & & \\
\hline 38 & Routine Assist. & $\mathrm{S}$ & $\mathrm{N}$ & MIN & GO & NEMO & DEMB & $\mathrm{T}$ & $\mathrm{F}$ & IND & SING & CTRL & INFO \\
\hline 39 & Routine Assist. & $\mathrm{S}$ & $\mathrm{N}$ & MIN & GO & NEMO & DEMB & $\mathrm{T}$ & $\mathrm{F}$ & TEAM & SING & PROD & INFO \\
\hline 40 & Routine Assist. & $\mathrm{S}$ & $\mathrm{N}$ & MIN & GO & NEMO & DEMB & - & $\mathrm{F}$ & IND & SING & PROD & MW \\
\hline 41 & Virtual Colleague & $\mathrm{S}$ & $\mathrm{N}$ & - & GO & NEMO & EMB & $\mathrm{S}$ & $\mathrm{P}$ & IND & SING & PROD & PRO \\
\hline 42 & Virtual Colleague & $\mathrm{N}$ & $\mathrm{B}$ & - & GO & - & DEMB & $\mathrm{T}$ & $\mathrm{P}$ & IND & SING & PROD & INFO \\
\hline \begin{tabular}{|l|l|}
43 & 44 \\
\end{tabular} & Virtual Colleague & $\mathrm{D}$ & B & MAX & GO & EMO & - & $\mathrm{S}$ & $\mathrm{P}$ & IND & $\begin{array}{l}\text { SING, } \\
\text { MULT }\end{array}$ & WENV & MW \\
\hline 45 & Virtual Colleague & $\mathrm{S}$ & B & MIN & GO & NEMO & DEMB & S & $\mathrm{F} / \mathrm{P}$ & IND & SING & WENV & MW \\
\hline 46 & Work Environ. & $\mathrm{S}$ & $\mathrm{N}$ & - & GO & NEMO & DEMB & $\mathrm{T}, \mathrm{S}$ & $\mathrm{F}$ & IND & SING & $\begin{array}{l}\text { PROD, } \\
\text { JOB }\end{array}$ & PRO \\
\hline \begin{tabular}{|l|l|}
477 & 48 \\
\end{tabular} & Work Environ. & $\mathrm{S}$ & $\mathrm{N}$ & - & GO & NEMO & DEMB & $\mathrm{T}, \mathrm{S}$ & $\mathrm{F}$ & IND & SING & $\begin{array}{l}\text { PROD, } \\
\text { WENV }\end{array}$ & INFO \\
\hline 49 & Decision Support & $\mathrm{S}$ & $\mathrm{N}$ & MIN & GO & NEMO & DEMB & $\mathrm{T}$ & $\mathrm{F}$ & TEAM & SING & DECM & INFO \\
\hline \begin{tabular}{|l|l|}
50 & 51 \\
\end{tabular} & Decision Support & $\mathrm{D}$ & $\mathrm{N}$ & - & GO & NEMO & DEMB & - & $\mathrm{E}$ & IND & MULT & DECM & $\begin{array}{c}\text { MAN, } \\
\text { PRO }\end{array}$ \\
\hline 52 & Reflection & $\mathrm{S}$ & $\mathrm{N}$ & MAX & GO & NEMO & DEMB & $\mathrm{T}, \mathrm{S}$ & $\mathrm{F}$ & IND & SING & WENV & ALL \\
\hline 53 & Reflection & $\mathrm{S}$ & $\mathrm{N}$ & MIN & GO & NEMO & DEMB & $\mathrm{T}$ & $\mathrm{F}$ & IND & SING & CTRL & INFO \\
\hline 54 & Team Creation & $\mathrm{S}$ & $\mathrm{N}$ & MIN & GO & NEMO & DEMB & $\mathrm{T}$ & $\mathrm{P}$ & IND & SING & PROD & INFO \\
\hline \multicolumn{14}{|c|}{$\begin{array}{l}\text { Legend: DD - Domain Depth: D - Deep, S - Shallow } \bullet \text { DB - Domain Breadth: N - Narrow, B - Broad } \bullet \text { SL - Session Length: MIN - Minimal, MAX - Maximal } \bullet \text { M } \\
\text { GD - Goal Direction: GO - Goal-Oriented, NGO - Non-Goal-Oriented } \bullet \text { EMO - Emotionality: EMO - Emotional, NEMO - Non-Emotional } \bullet \text { REP - Representation: } \\
\text { EMB - Embodied, DEMB - Disembodied } \bullet \text { MOD - Modalitites: T - Text, S - Speech, H - Haptic } \bullet \text { RO - Role: F - Facilitator, P - Peer, E - Expert } \bullet \text { NH - No. of } \\
\text { Humans: IND - Individual, TEAM - Team, CROWD - Crowd } \bullet \text { NCA - No. of CAs: SING - Single, MULT - Multiple } \bullet \text { IMP - Impact: DECM - Decision Making, } \\
\text { CTRL - Control, PROD - Productivity, SI - Social Interaction, JOB - Job Enhancement, WENV - Work Environment } \bullet \text { WO - Type of Worker: MAN - Managers, } \\
\text { PRO - Professionals, C/T - Clericals/Technicians, MW - Manual Worker, ALL - All }\end{array}$} \\
\hline
\end{tabular}

Table 2. Classfication of WCAs for workers. Dimensions are abbreviated as introduced in Section 4.2. 
staff. A similar constellation of user and impacted persons can be observed in the application domain of teaching, coaching and tutoring. The usage of CAs as a virtual teacher [67], to support peer learning and knowledge building [68] and as a learning partner [69] were proposed in the publications in our result set.

WCAs for non-workers that impact a workplace are also used for automation purposes. This includes the virtualization of service roles, such as pre- and post-sales customer service [70, 71, 72, 73], in information providing services [74], airport security [75] and tech support desks [76]. While the earlier application domains mainly use WCAs as a supportive tool to relieve the workers impacted, automation may pose a threat to the respective jobs as they may make the human-added value negligible.

\subsection{Design Aspects of WWCAs}

Several of the above mentioned WWCAs have been evaluated in studies to inform the design of such CAs. Therefore, we summarize the findings from the respective studies to extract aspects that require special attention when designing a WWCA. These aspects, however, need to be tested in further evaluations in order to formulate generalizable design principles. Also, we do not claim these aspects to be WWCA-specific as most of these apply to any CAs, or system at the workplace.

Design for Diversity. Diversity is present in different forms at the workplace, including differently enabled people. With the flexibility of modalities for WWCAs, it can be advantageous to provide multiple, concurrent modalities (e.g. speech, text), different representations of the same information (e.g. visual, textual) [35] or different levels of detail [24]. Clear instructions and clear turn-taking mechanisms should be implemented as well as resilient repair strategies in case of a breakdown to fool-proof the CA for non-tech-savvy users [29]. The workplace needs to be taken into consideration, workers in noisy environments (e.g. lab) can hardly use audio-based CAs [28], while the farmers using FarmChat [35] had a hard time seeing displayed information in the direct sunlight.

Efficient Modalities. Time at the workplace is costly, therefore, an efficient modality should be selected for the WWCA. Text has proven to be faster and clearer, while audio is better suitable for illiterate users and provides a more natural and anthropomorphized way of communication with the CA [35]. In the workplace context, some tasks are perceived differently depending on the input medium, e.g. journaling feels more like a formal report when actually spoken [52]. It should be taken into consideration that speech can be overheard by surrounding people which, in most cases, is not a desired effect due to privacy concerns [52] or competition among peers [35]. As of the WWCAs in our SLR, many of the CAs make use of already existing communication means in the digital workplace, e.g. Slack, Skype, email or other Instant Messengers [39, 52. 31, 53, 36, 77]. This acts as a preliminary ensurance that the user is already familiar with the basic functions of the application.

Privacy and Data Protection. With the workplace being a sensitive environment, it is important to ensure conversations with the WWCA to be as private and anonymous as possible. This applies to handling with sensitive topics like bullying and harassment [29] as well as any personal information like individual availability and appointments [36], personal thoughts [52] or logs of individual behavior at work [53]. It can also help to be transparent to the user about the personal data stored [53].

Reliability and Resilience. At the workplace, system performance is a strong predictor for user satisfaction, even more than the friendliness of the CA [31]. The user has to trust the CA as stakes are usually high at the workplace [35, 41]. In case of a breakdown, the CA should fail gracefully [39] rather than doing "the next best thing" to maintain the user's trust and the façade of a properly running system.

Proactivity and Risk of Interruption. WWCAs are often designed to be capable of being proactive [42]. Therefore, the value of the interruption and the perceived severity of interruption needs to be precisely balanced [31, 53]. Using the user's current context, e.g. through calendars or using ubiquitous devices [78], the cost of interruption can be decreased by choosing low-cost moments [31, 39].

Mental Model. The mental model that the user creates in his mind influences the degree of the user's acceptance. Tseng et al. [53] report that some participants refused to negotiate with the CA because they perceived it as a machine rather than a conscious being worth bargaining with.

Implications on the Workplace. Highly anthropomatic, assistive WWCAs may appear to be human to strangers. This may elevate the user's apparent hierarchical position, since having an assistant is often an indicator for superiority [36], which is an effect that is sometimes unwanted (e.g. when communicating to the manager). WWCAs also bear the risk of eliminating human interactions that are vital in team work [36] or over-controlling workers to a level where they lose their own sense of control [53]. 


\section{Discussion}

We structure our discussion as follows: First, we describe the limitations to this study, then, we point out observations we made during the analysis of the publications. Lastly, we discuss the implication for further research and propose research questions as starting points.

\subsection{Limitations}

There are several limitations to this work: Due to our selection of data sources, we do not claim that our SLR is exhaustive. In particular, this topic has proven to be a very recent item of study and most probably still part of ongoing, unpublished research. The literature included in this work are primarily retrieved in April/May 2019 with minor additions in September 2019. Also, we only included literature from 2010 or newer due to the increased interest in this topic in the recent years. This inclusion criteria may have created a recency bias in our results. The design aspects we extracted originated from publications with empirical evaluations of their concepts with no further classification of their quality. Thus, the aspects only rely on the quality of its respective source and were not re-evaluated in the scope of this work. As mentioned in Section 4.1 the majority of publications had affiliations to an institution in the U.S. and the U.K. Therefore, our results may only reflect concepts and design aspects conceivable and important to the Western culture and the Western way of working. Lastly, we only included WWCA concepts where the CA is the main contribution, i.e. concepts including a physical embodiment (robot) were excluded.

\subsection{Observations}

Our main observation for WWCAs are apparent in Table 2. In the workplace context, CAs to this day are primarily used for purposes that do not require many human characteristics besides of a name, i.e. almost none of the WWCAs are emotional or have an anthropomorphised embodiment. This may be reasoned by the primary impact of improvement in productivity which can be optimally achieved with pragmatic systems with little conversational overhead. Same reason could explain the fact that text input is the most prevalent modality for input and output. Text can be read in a faster, individual pace or be only scanned while speech becomes inaudible with increasing speed. Furthermore, most of the WWCA concepts we found are designed to be used in an office, thus targets at information workers. In an office, speech input and output may not be suitable as explained in Section 4.5

\subsection{Implications and Further Research}

The majority of research on this topic originate from English-speaking, "Western" countries. This raises the question whether there are culture-bound differences, e.g. in preferred modality, WWCA personality or generally the acceptance of a CA at the workplace. We expect that with the on-going advancements in NLU for other languages, the usage of a WWCA becomes more conceivable in the near future for other cultures and would propose the investigations of parallels and differences in design of such in comparison to the previously presented.

We have extracted several design aspects to be taken into consideration from our result set. However, concrete design principles need to be developed, instantiated and validated in more studies, including different industries, application domains, countries, roles and (company) cultures. We expect design principles for WWCA to be culture-bound and differ depending on application domain and workplace.

As mentioned in our observations, almost all of the WWCAs retrieved in our SLR target information workers. Certainly, it is more conceivable to integrate a CA into the workplace due to the existing technology at an office workplace, however, as proven by FarmChat [35], WWCAs can also be of high value to low-tech and manual workers. Therefore, we encourage future research to explore the transferability of existing application domains for manual workers, or define new applications domains specific to manual workers.

\section{Conclusion}

Our SLR revealed that there are two substantially different types of CAs at the workplace: Namely, those facing the worker and those facing a non-working person, yet having a great impact on the workplace. We have identified 29 CAs for the workplace and for working users (WWCAs) which contribute to eight different application domains, ranging from a working tool with little anthropomorphism to a human-like virtual colleague. From these WWCAs, we extracted seven aspects that need to be particularly considered when designing a WWCA, however, it is yet to be investigated in further research as the number of studies performed on the WWCAs is still relatively small. Nonetheless, our SLR supported the claim that WCAs are emerging quickly [5] and should be an item of interest in further CA research. 


\section{Acknowledgements}

This work was supported by the Federal Ministry of Labour and Social Affairs of Germany (Bundesministerium für Arbeit und Soziales) as part of the research project Kompetenzen entwickeln und richtig nutzen (KERN) within the initiative Initiative Neue Qualität der Arbeit (INQA).

\section{References}

[1] G. Oswald, "Star Trek Voice First Computer," 1966. Available at: https://youtu.be/1ZXugicgn6U (accessed June 06, 2019).

[2] J. Weizenbaum, "ELIZA - A Computer Program For the Study of Natural Language Communication Between Man And Machine," Computational Linguistics, vol. 9, pp. 36-45, Jan. 1966.

[3] K. M. Colby, Artificial Paranoia: A Computer Simulation of Paranoid Process. Pergamon Press, 1975.

[4] S. Stieglitz, F. Brachten, and T. Kissmer, "Defining Bots in an Enterprise Context," in Proceedings of the 2018 International Conference on Information Systems, 2018.

[5] R. Meyer von Wolff, S. Hobert, and M. Schumann, "How May I Help You? - State of the Art and Open Research Questions for Chatbots at the Digital Workplace," in Proceedings of the 52nd Hawaii International Conference on System Sciences, 2019.

[6] J. Webster and R. T. Watson, "Analyzing the Past to Prepare for the Future: Writing a Literature Review," MIS Quarterly, pp. xiii-xxiii, 2002.

[7] J. C. Lester, K. Branting, and B. W. Mott, "Conversational Agents," in The Practical Handbook of Internet Computing., 2004.

[8] J. Grudin and R. Jacques, "Chatbots, Humbots, and the Quest for Artificial General Intelligence," in Proceedings of the 2019 CHI Conference on Human Factors in Computing Systems, 2019.

[9] A. Ram, R. Prasad, C. Khatri, A. Venkatesh, R. Gabriel, Q. Liu, J. Nunn, B. Hedayatnia, M. Cheng, A. Nagar, et al., "Conversational AI: The Science Behind the Alexa Prize," arXiv preprint arXiv:1801.03604, 2018.

[10] M. Mitrevski, "Getting Started with Wit.ai," in Developing Conversational Interfaces for iOS, pp. 143-164, Berkeley, CA: Apress, 2018.

[11] L. C. Klopfenstein, S. Delpriori, S. Malatini, and A. Bogliolo, "The Rise of Bots: A Survey of Conversational Interfaces, Patterns, and Paradigms," in Proceedings of the 2017 Conference on Designing Interactive Systems, pp. 555-565, 2017.

[12] A. Turing, "Computing Machinery and Intelligence," MIND - A Quarterly Review of Psychology and Philosophy, vol. 59, pp. 433-460, Oct. 1950.

[13] N. Block, "The Turing Test: Verbal Behavior as the Hallmark of Intelligence," 1981.

[14] H. N. Io and C. B. Lee, "Chatbots and Conversational Agents: A Bibliometric Analysis," in Proceedings of the 2017 IEEE International Conference on Industrial Engineering and Engineering Management, pp. 215-219, 2017.
[15] K. K. Fitzpatrick, A. Darcy, and M. Vierhile, "Delivering Cognitive Behavior Therapy to Young Adults With Symptoms of Depression and Anxiety Using a Fully Automated Conversational Agent (Woebot): A Randomized Controlled Trial," JMIR Mental Health, vol. 4, no. 2, p. e19, 2017.

[16] B. Inkster, S. Sarda, and V. Subramanian, "An Empathy-Driven, Conversational Artificial Intelligence Agent (Wysa) for Digital Mental Well-Being: Real-World Data Evaluation Mixed-Methods Study," JMIR mHealth and uHealth, vol. 6, no. 11, p. e12106, 2018.

[17] U. Gnewuch, S. Morana, and A. Mädche, "Towards Designing Cooperative and Social Conversational Agents for Customer Service," in Proceedings of the 2017 International Conference on Information Systems, 2017.

[18] E. Bittner, S. Oeste-Reiß, and J. M. Leimeister, "Where Is The Bot in Our Team? Toward a Taxonomy of Design Option Combinations for Conversational Agents in Collaborative Work," in Proceedings of the 52nd Hawaii International Conference on System Sciences, 2019.

[19] H.-Y. Shum, X.-d. He, and D. Li, "From Eliza to XiaoIce: Challenges and Opportunities with Social Chatbots," Frontiers of Information Technology \& Electronic Engineering, vol. 19, no. 1, pp. 10-26, 2018.

[20] J. Vom Brocke, A. Simons, B. Niehaves, K. Riemer, R. Plattfaut, and A. Cleven, "Reconstructing the Giant: On the Importance of Rigour in Documenting the Literature Search Process," in Proceedings of the 2019 European Conference on Information Systems, pp. 2206-2217, 2009.

[21] K. L. Kraemer and J. N. Danziger, "The Impacts of Computer Technology on the Worklife of Information Workers," Social Science Computer Review, vol. 8, no. 4, pp. 592-613, 1990.

[22] R. J. L. John, N. Potti, and J. M. Patel, "Ava: From Data to Insights Through Conversations.", in Proceedings of the 8th Biennial Conference on Innovative Data Systems Research, 2017.

[23] E. Fast, B. Chen, J. Mendelsohn, J. Bassen, and M. S. Bernstein, "Iris: A Conversational Agent for Complex Tasks," in Proceedings of the 2018 CHI Conference on Human Factors in Computing Systems, pp. 1-12, 2018.

[24] A. Abdellatif and E. Shihab, "MSRBot: Using Bots to Answer Questions from Software Repositories," arXiv preprint arXiv:1905.06991, 2019.

[25] Z. Xue, T.-Y. Ko, N. Yuchen, M.-K. D. Wu, and C.-C. Hsieh, "Isa: Intuit Smart Agent, A Neural-Based Agent-Assist Chatbot," in Proceedings of the 2018 IEEE International Conference on Data Mining Workshops, pp. 1423-1428, 2018.

[26] A. A. Chandrashekara, R. K. M. Talluri, S. S Sivarathri, R. Mitra, P. Calyam, K. Kee, and S. Nair, "Fuzzy-based Conversational Recommender for Data-Intensive Science Gateway Applications," in Proceedings of the 2018 IEEE International Conference on Big Data, pp. 4870-4875, 2018.

[27] P. K. Chilana, N. Hudson, S. Bhaduri, P. Shashikumar, and S. Kane, "Supporting Remote Real-Time Expert Help: Opportunities and Challenges for Novice 3D Modelers," in Proceedings of the 2018 IEEE Symposium on Visual Languages and Human-Centric Computing, pp. 157-166, 2018. 
[28] J. Cambre, Y. Liu, R. E. Taylor, and C. Kulkarni, "Vitro: Designing a Voice Assistant for the Scientific Lab Workplace," in Proceedings of the 2019 Conference on Designing Interactive Systems, pp. 1531-1542, 2019.

[29] A. Latham, K. Crockett, and Z. Bandar, "A Conversational Expert System Supporting Bullying and Harassment Policies," in Proceedings of the 2nd International Conference on Agents and Artificial Intelligence, pp. 163-168, 2010.

[30] K. Crockett, J. O‘Shea, and Z. Bandar, "Goal Orientated Conversational Agents: Applications to Benefit Society," in Agent and Multi-Agent Systems: Technologies and Applications, vol. 6682, pp. 16-25, 2011.

[31] Q. V. Liao, M. Davis, W. Geyer, M. Muller, and N. S. Shami, "What Can You Do? Studying Social-Agent Orientation and Agent Proactive Interactions with an Agent for Employees," in Proceedings of the 2016 Conference on Designing Interactive Systems, 2016.

[32] R. Y. M. Li, "Software Engineering and Reducing Construction Fatalities: An Example of the Use of Chatbot," in An Economic Analysis on Automated Construction Safety, pp. 105-116, 2018.

[33] C. E. Kaed, A. Ponnouradjane, and D. Shah, "A Semantic Based Multi-Platform IoT Integration Approach from Sensors to Chatbots," in Proceedings of the 2018 Global Internet of Things Summit, pp. 1-6, 2018.

[34] A. Piyatumrong, C. Sangkeettrakarn, S. Witdumrong, and J. Cherdgone, "Chatbot Technology Adaptation to Reduce the Information Gap in R\&D Center: A Case Study of an IT Research Organization," in Proceedings of the 2018 Portland International Conference on Management of Engineering and Technology, pp. 1-9, 2018.

[35] M. Jain, P. Kumar, I. Bhansali, Q. V. Liao, K. Truong, and S. Patel, "FarmChat: A Conversational Agent to Answer Farmer Queries," Proceedings of the ACM on Interactive, Mobile, Wearable and Ubiquitous Technologies, vol. 2, no. 4, 2018.

[36] J. Cranshaw, E. Elwany, T. Newman, R. Kocielnik, B. Yu, S. Soni, J. Teevan, and A. Monroy-Hernández, "Calendar.help: Designing a Workflow-Based Scheduling Agent with Humans in the Loop," in Proceedings of the 2017 CHI Conference on Human Factors in Computing Systems, pp. 2382-2393, 2017.

[37] E. Mulyana, R. Hakimi, and Hendrawan, "Bringing Automation to the Classroom: A ChatOps-Based Approach," in Proceedings of the 4th International Conference on Wireless and Telematics, pp. 1-6, 2018.

[38] D. Ramasubbu, K. Baskaran, and G. Yann, "Intrusive Plug Management System Using Chatbots in Office Environments," in Proceedings of the 2018 Asian Conference on Energy, Power and Transportation Electrification, pp. 1-4, 2018.

[39] C. Toxtli, A. Monroy-Hernández, and J. Cranshaw, "Understanding Chatbot-mediated Task Management," in Proceedings of the 2018 CHI Conference on Human Factors in Computing Systems, pp. 1-6, 2018.

[40] R. Y. M. Li, "Automated and Intelligent Tools in the Construction Industry," in Construction Safety Informatics, pp. 103-119, 2019.

[41] H. Trinh, L. Ring, and T. Bickmore, "DynamicDuo: Co-Presenting with Virtual Agents," in Proceedings of the 2015 CHI Conference on Human Factors in Computing Systems, pp. 1739-1748, 2015.
[42] P. Chandar, Y. Khazaeni, M. Davis, M. Muller, M. Crasso, Q. V. Liao, N. S. Shami, and W. Geyer, "Leveraging Conversational Systems to Assists New Hires During Onboarding," in Human-Computer Interaction - INTERACT 2017, vol. 10514 of Lecture Notes in Computer Science, 2017.

[43] J. Fank, N. T. Richardson, and F. Diermeyer, "Anthropomorphising Driver-Truck Interaction: A Study on the Current State of Research and the Introduction of Two Innovative Concepts," Journal on Multimodal User Interfaces, 2019.

[44] J. Fank and M. Lienkamp, ““I'm your personal Co-Driver-How can I assist you?" Assessing the Potential of Personal Assistants for Truck Drivers," in Intelligent Human Systems Integration 2019, pp. 795-800, 2019.

[45] D. R. Large, G. Burnett, V. Antrobus, and L. Skrypchuk, "Stimulating conversation: Engaging Drivers in Natural Language Interactions with an Autonomous Digital Driving Assistant to Counteract Passive Task-Related Fatigue," in International Conference on Driver Distraction and Inattention, 2017.

[46] P. Mavridis, O. Huang, S. Qiu, U. Gadiraju, and A. Bozzon, "Chatterbox: Conversational Interfaces for Microtask Crowdsourcing," in Proceedings of the 27th ACM Conference on User Modeling, Adaptation and Personalization, 2019.

[47] W. S. Lasecki, R. Wesley, J. Nichols, A. Kulkarni, J. F. Allen, and J. P. Bigham, "Chorus: A Crowd-powered Conversational Assistant," in Proceedings of the 26th Annual ACM Symposium on User Interface Software and Technology, pp. 151-162, 2013.

[48] W. S. Lasecki, P. Thiha, Y. Zhong, E. Brady, and J. P. Bigham, "Answering Visual Questions with Conversational Crowd Assistants," in Proceedings of the 15th International Conference on Computers and Accessibility, pp. 18:1-18:8, 2013.

[49] N. Terzimehić, M. Khamis, F. Bemmann, and H. Hussmann, "Lunchocracy: Improving Eating Dynamics in the Workplace Using a Bot-Based Anonymous Voting System," in Extended Abstracts of the 2018 CHI Conference on Human Factors in Computing Systems, pp. LBW022:1-LBW022:6, 2018.

[50] A. Augello, G. Pilato, and S. Gaglio, "An Intelligent Advisor to Suggest Strategies in Economic Policy Decisions," in Proceedings of the 2010 International Conference on Complex, Intelligent and Software Intensive Systems, pp. 734-739, 2010.

[51] A. Augello, G. Pilato, and S. Gaglio, "Intelligent Advisor Agents in Distributed Environments," in Information Retrieval and Mining in Distributed Environments, vol. 324, pp. 109-124, 2010.

[52] R. Kocielnik, D. Avrahami, J. Marlow, D. Lu, and G. Hsieh, "Designing for Workplace Reflection: A Chat and Voice-Based Conversational Agent," in Proceedings of the 2018 Designing Interactive Systems Conference, pp. 881-894, 2018.

[53] V. W.-S. Tseng, M. L. Lee, L. Denoue, and D. Avrahami, "Overcoming Distractions during Transitions from Break to Work using a Conversational Website-Blocking System," in Proceedings of the 2019 CHI Conference on Human Factors in Computing Systems, 2019.

[54] Z. Xiao, M. X. Zhou, and W.-T. Fu, "Who Should Be My Teammates: Using a Conversational Agent 
to Understand Individuals and Help Teaming," in Proceedings of the 24th International Conference on Intelligent User Interfaces, pp. 437-447, 2019.

[55] T. W. Bickmore, S. E. Mitchell, B. W. Jack, M. K. Paasche-Orlow, L. M. Pfeifer, and J. O'Donnell, "Response to a relational agent by hospital patients with depressive symptoms," Interacting with Computers, vol. 22, no. 4, pp. 289-298, 2010.

[56] T. Bickmore, L. Vardoulakis, B. Jack, and M. Paasche-Orlow, "Automated Promotion of Technology Acceptance by Clinicians Using Relational Agents," in Intelligent Virtual Agents, vol. 8108, pp. 68-78, 2013.

[57] T. Shinozaki, E. Bissay, Y. Ikegami, and S. Tsuruta, "Applying Context Respectful Summarization to Counseling Agent for the Japanese," in Proceedings of the 2013 International Conference on Signal-Image Technology \& Internet-Based Systems, pp. 916-923, 2013.

[58] G. Cameron, D. Cameron, G. Megaw, R. Bond, M. Mulvenna, S. O'Neill, C. Armour, and M. McTear, "Towards a Chatbot for Digital Counselling," in Proceedings of the 31 st British Computer Society Human Computer Interaction Conference, pp. 24:1-24:7, BCS Learning \& Development Ltd., 2017.

[59] A. Yorita, S. Egerton, J. Oakman, C. Chan, and N. Kubota, "A Robot Assisted Stress Management Framework: Using Conversation to Measure Occupational Stress," in Proceedings of the 2018 IEEE International Conference on Systems, Man, and Cybernetics, pp. 3761-3767, Oct. 2018.

[60] J. Ren, T. Bickmore, M. Hempstead, and B. Jack, "Birth Control, Drug Abuse, or Domestic Violence? What Health Risk Topics Are Women Willing to Discuss with a Virtual Agent?," in Intelligent Virtual Agents, vol. 8637, pp. 350-359, 2014.

[61] A. Bresó, J. Martínez-Miranda, C. Botella, R. M. Baños, and J. M. García-Gómez, "Usability and Acceptability Assessment of an Empathic Virtual Agent to Prevent Major Depression," Expert Systems, vol. 33, no. 4, pp. 297-312, 2016.

[62] J. Martínez-Miranda, "Embodied Conversational Agents for the Detection and Prevention of Suicidal Behaviour: Current Applications and Open Challenges," Journal of Medical Systems, vol. 41, no. 9, 2017.

[63] R. Kocielnik, G. Hsieh, and D. Avrahami, "Helping Users Reflect on Their Own Health-Related Behaviors," in Studies in Conversational UX Design, pp. 85-115, 2018.

[64] J. Pereira and Ó. Díaz, "Using Health Chatbots for Behavior Change: A Mapping Study," Journal of Medical Systems, vol. 43, no. 5, 2019.

[65] B. Fielding, P. Kinghorn, K. Mistry, and L. Zhang, "An Enhanced Intelligent Agent with Image Description Generation," in Intelligent Virtual Agents, vol. 10011, pp. 110-119, 2016.

[66] J. Sin and C. Munteanu, "A Preliminary Investigation of the Role of Anthropomorphism in Designing Telehealth Bots for Older Adults," in Extended Abstracts of the 2019 CHI Conference on Human Factors in Computing Systems, pp. LBW1820:1-LBW1820:6, 2019.

[67] A. Silvervarg and A. Jönsson, "Subjective and objective evaluation of conversational agents in learning environments for young teenagers," in Proceedings of the 7th IJCAI Workshop on Knowledge and Reasoning in Practical Dialogue Systems, 2011.
[68] S. Tegos and S. N. Demetriadis, "Conversational Agents Improve Peer Learning through Building on Prior Knowledge," Educational Technology \& Society, vol. 20, pp. 99-111, 2017.

[69] A. Ogan, S. Finkelstein, E. Mayfield, C. D’Adamo, N. Matsuda, and J. Cassell, “'”oh Dear Stacy!”: Social Interaction, Elaboration, and Learning with Teachable Agents," in Proceedings of the 2012 CHI Conference on Human Factors in Computing Systems, pp. 39-48, 2012.

[70] M. Kaleem, J. O'Shea, and K. Crockett, "Development of Umair the Urdu Conversational Agent for Customer Service," in Proceedings of the World Congress on Engineering, 2014.

[71] A. Xu, Z. Liu, Y. Guo, V. Sinha, and R. Akkiraju, "A New Chatbot for Customer Service on Social Media," in Proceedings of the 2017 CHI Conference on Human Factors in Computing Systems, pp. 3506-3510, 2017.

[72] T. Hu, A. Xu, Z. Liu, Q. You, Y. Guo, V. Sinha, J. Luo, and R. Akkiraju, "Touch Your Heart: A Tone-aware Chatbot for Customer Care on Social Media," in Proceedings of the 2018 CHI Conference on Human Factors in Computing Systems, pp. 1-12, 2018.

[73] D. Baier, A. Rese, and M. Räglinger, "Conversational User Interfaces for Online Shops? A Categorization of Use Cases," in Proceedings of the 2018 International Conference on Information Systems, 2018.

[74] M. Choque-Díaz, J. Armas-Aguirre, and P. Shiguihara-Juárez, "Cognitive Technology Model to Enhanced Academic Support Services with Chatbots," in Proceedings of the 2018 IEEE XXV International Conference on Electronics, Electrical Engineering and Computing, pp. 1-4, 2018. ISSN:.

[75] J. F. Nunamaker, D. C. Derrick, A. C. Elkins, J. K. Burgoon, and M. W. Patton, "Embodied Conversational Agent-Based Kiosk for Automated Interviewing," Journal of Management Information Systems, vol. 28, no. 1, pp. 17-48, 2011.

[76] A. A.-e. S. Ibrahim, "A Study About Using a Cognitive Agent in Replacing Level 1 and 2 Service Desk Activities," in Third International Congress on Information and Communication Technology, vol. 797, pp. 307-316, 2019.

[77] Q. V. Liao, W. Geyer, M. Mas-ud Hussain, P. Chandar, M. Davis, Y. Khazaeni, M. P. Crasso, D. Wang, M. Muller, and N. S. Shami, "All Work and No Play? Conversations with a Question-and-Answer Chatbot in the Wild," in Proceedings of the 2018 CHI Conference on Human Factors in Computing Systems, pp. 1-13, 2018.

[78] R. Rissler, M. Nadj, M. X. Li, M. T. Knierim, and A. Maedche, "Got Flow?: Using Machine Learning on Physiological Data to Classify Flow," in Extended Abstracts of the 2018 CHI Conference on Human Factors in Computing Systems, pp. LBW612:1-LBW612:6, 2018. 